significantly deviate from the principal stress directions. Thus, the neglect of friction in concrete is not on the side of safety.

This interesting result was reached only for membrane loading. It is our objective to develop the analogous frictional limit design method for the tensile reinforcement in plates under bending and twisting moments, possibly combined

\section{Concrete Plate Reinforcement: Frictional Limit Design}

\author{
By Zdenêk P. Bažant,' F. ASCE and Chuan Lin
}

Asemmact: The limit design of a regular rectangular reinforcing net in a concrete plate subjected to arbitrary normal and shear forces and bending and twisting moments is studied. The assumptions are: The reinforcement is plastic; concrete resists no tension; a normal crack of any direction may exist in concrete; the normal stress in concrete is constant; in-plane shear stresses can be transmitted in concrete, are of constant magnitude but possibly of alternating sign, and their ratio to the normal compressive stresses may not exceed the friction coefficient. The previously developed frictional (no-slip) limit design for membrane loading is the special case when the compression zone covers the whole thickness on plate. Design charts for he case or no membrane forces are computed and plotled. They differ up to about $20 \%$ from the classical solution. In contrast pure membrane loading, the frictional design for failure may yield a lighter reinforcement than the classical design. Mostly, hars cars are laid in the directier be $45^{\circ}$ for the optimum design.

\section{INTROouction}

This paper deals with the limit design of regular rectangular reinforcing nets for concrete plates subjected to membrane (in-plane) as well as flexural loading. This is a fundamental problem of reinforced concrete, and much has been already learned about it $(1,8-10,12-16,20-26)$. Of particular interest are the studies of Morley (22), Baumann (1), and Gupta (14) in which the current design approach is crystallized. A salient feature of this approach, which we will call "classical" or "frictionless," is the tacit assumption that the cracks in concrete cannot slip, i.e., friction cannot develop on the cracks. Recently, however, it has been discovered $(5,7)$ that the limit design in which friction is taken into account $(5,7,17,19)$ never yields a lighter reinforcement than the classical design, and may require a substantially heavier reinforcement if the directions of the bars

'Prof. of Civ. Engrg. and Dir., Center for Concrete and Geomaterials, Technological Inst., Northwestern Univ., Evanston, Ill. 60201.

Grad. Research Asst., Northwestern Univ., Evanston, III., 60201; presently Engr. Dynamic Load Evaluation Unit, Nuclear Energy Business Operation, General Electric Co., San Jose, Calif. 95125.

Note-Discussion open until April 1, 1983. To extend the closing date one month, a written request must be filed with the Manager of Technical and Professional Publications, ASCE. Manuscript was submitted for review for possible publication on October 6, 1981 This paper is part of the Journal of the Structural Division, Proceedings of the American Society of Civil Engineers, OASCE, Vol. 108, No. STI1, November, 1982. ISSN 00448001/82/0011-2443/\$01.00. also with in-plane normal and shear forces. As we will see, consideration of friction is again important.

According to a recent study (4), the use of a frictional design for membrane loading limits deformations and crack width when the principal internal forces form large angles with all reinforcing bars. The same effect could be expected for bending, but we cannot study it here.

\section{Basic Assumptions}

We consider that the plate is reinforced by a regular orthogonal grid of steel bars near the bottom surface (Fig. 1), and we assume that the spacing of the bars is sufficiently dense to allow treating the grid as a continuous layer and considering the internal forces to be constant over a distance of several bar spacings. For the sake of simplicity, and because its effect is small, we do not consider a second reinforcing grid in the compression zone, although our method of analysis could cope with it also. We assume the concrete to be cracked in a plane normal to the plate. The crack extends through part of the thickness.

As the basic principle of design, we assume that, regardless of the given loading, a crack of any orientation and any depth may be present. ("If anything can go wrong it will," Murphy's Law.) In the same spirit, the upper bound theorem of limit analysis requires us to consider all possible collapse mechanisms, and each crack may be regarded as one mechanism.

First, we need to decide on proper and sufficiently simple assumptions about the stresses in concrete at the crack plane. As for the normal stresses in concrete, $\sigma_{n}$, we adopt the usual assumption that they are constant over the entire depth $a$ of the compression zone (Fig. 1) and that no tensile stresses can be transmitted by concrete. As for the shear stresses, $\sigma_{n,}$, in concrete, only crack directions for which these shear stresses are insignificant are considered in the classical design approach. We will, however, consider cracks of any direction, and then significant shear stresses, $\sigma_{n e}$, may arise for some directions in the compression zone. What should we assume about their distribution?

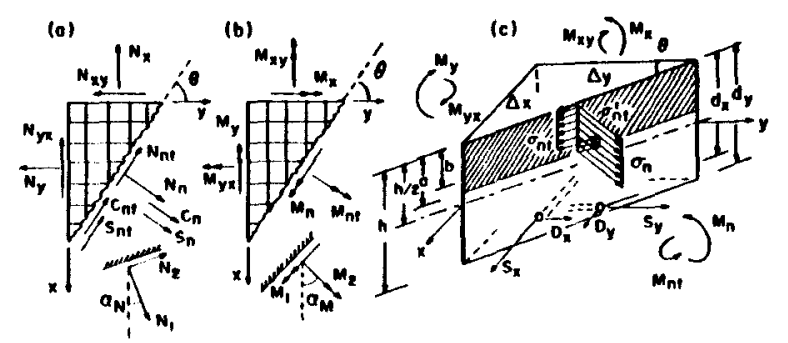

FIG. 1.-Internal Forces, Notations, and Deformation Mode 
The shear stresses in concrete obviously cannot be arbitrary. There must be a limit on $\sigma_{m}$ for which we will later introduce the concept of friction (Eqs. 6 and 13). Since $\sigma_{n}$ is assumed to be distributed uniformly, it is now reasonable (and consistent with the concept of friction) to assume that the magnitude of $\sigma_{m}$ is constant over the entire compression zone, but the sign of $\sigma_{n t}$ may change at a certain depth, $b(b \leq a$, see Fig. $1(c))$, so that the compression zone alone may transmit some torque. The case when $\sigma_{n}$ is of one sign is then the special case for $b=a$.

Due to aggregate interlock, membrane shear stresses can also exist on the crack outside the uncracked compression zone. Shear stresses on the crack cannot exist, however, without normal compressive stresses (2), and so the portion of the crack transmitting membrane frictional shear stresses would have to be counted to the compression zone. Although the values of $\sigma_{n}$ and $\sigma_{n t}$ on the crack would presumably be less than in the uncracked compression zone, simplicity requires us to consider a uniform distribution of $\sigma_{n}$, and, consequently, also of $\sigma_{m}$. Since $\sigma_{n}$ must act over the same area as $\sigma_{n}$, we then conclude that the frictional shear stresses on the crack outside the compression zone should be considered zero.

There is another reason for assuming zero membrane shear stresses on the crack outside the compression zone. It has to do with the magnitude of the slip (relative tangential displacement) on the crack. Frictional shear stresses are produced only after a sufficient slip, depending on the opening width of the crack (2). In case of a through-crack (membrane loading), a sufficient slip can occur to mobilize the frictional stresses. However, for a crack through part of the thickness, a sufficient slip probably cannot occur due to the stiffness resulting from contiguity of concrete within the compression zone.

The recently developed frictional (no-slip) limit design for membrane loading $(5,7)$ may be formally obtained as the special case of our model when what we call the "compression zone" covers the entire thickness of the plate $(a=b=$ $h$ ). The compression zone must then be physically interpreted as a crack with aggregate interlock.

The case $a=0$ implies zero shear stresses in concrete, and cannot represent the frictional limit design; it yields, however, the classical frictionless design $(1,10)$ for tensile membrane loading. This design is also obtained for $a=b=$ $h$ when the friction coefficient tends to infinity $(5,7)$.

\section{Equilubaum and Ineoualuty Condinons}

The internal forces in the plate may be characterized by normal forces, $N_{x}$, $N_{y}$ (positive if tensile), shear forces, $N_{x y}$, bending moments, $M_{x}, M_{y}$, and twisting moments, $M_{x y}$ (Fig. 1). The signs are shown in Fig. 1 where double arrows denote the axial vectors of moments, which turn counterclockwise when looking against the arrow. In terms of the principal normal forces, $N_{1}, N_{2}$, and the principal bending moments, $M_{1}, M_{2}$, the angles of which with axis $x$ are $\alpha_{N}$ and $\alpha_{M}$, respectively, we may write

$N_{x}=N_{1} \cos ^{2} \alpha_{N}+N_{2} \sin ^{2} \alpha_{N} ; \quad N_{y}=N_{1} \sin ^{2} \alpha_{N}+N_{2} \cos ^{2} \alpha_{N} ;$

$N_{x y}=\left(N_{2}-N_{1}\right) \sin \alpha_{N} \cos \alpha_{N} ; \quad M_{x}=M_{1} \cos ^{2} \alpha_{M}+M_{2} \sin ^{2} \alpha_{M} ;$

$M_{y}=M_{1} \sin ^{2} \alpha_{M}+M_{2} \cos ^{2} \alpha_{M} ; \quad M_{x y}=\left(M_{2}-M_{1}\right) \sin \alpha_{M} \cos \alpha_{M} \ldots \ldots \ldots$
The normal and shear forces, and the bending and twisting moments on the plane of the crack (Fig. 1) forming angle $\theta$ with axis $y$ are

$N_{n}=N_{x} C^{2}+N_{y} S^{2}+2 N_{x y} S C ; \quad N_{n t}=\left(N_{y}-N_{x}\right) S C+N_{x y}\left(C^{2}-S^{2}\right)$

$M_{n}=M_{x} C^{2}+M_{y} S^{2}+2 M_{x y} S C ; \quad M_{n t}=\left(M_{y}-M_{x}\right) S C+M_{x y}\left(C^{2}-S^{2}\right)$.

in which $C=\cos \theta$; and $S=\sin \theta$.

If we further denote by $S_{x}$ and $S_{y}$ the axial yield forces in the reinforcement (per unit length of plate), and by $D_{x}, D_{y}$, the dowel forces, i.e., the shear forces transmitted by the bars across the crack plane (normal to $S_{x}$ and $S_{y}$ ), the resultants that the yield forces in reinforcement have in the normal and tangential directions to the crack plane are

$S_{n}=S_{x}\left(C^{2}+\delta_{1} S^{2}\right)+S_{y}\left(S^{2}+\delta_{2} C^{2}\right) ; \quad S_{n t}=\left[\left(1-\delta_{2}\right) S_{y}-\left(1-\delta_{1}\right) S_{x}\right] S C$

in which $\delta_{1}=D_{x} / S_{x}$ and $\delta_{2}=D_{y} / S_{y}$ are the dowel force coefficients for the $x$ and $y$ directions, respectively. They are assumed to be given.

Summing the forces and the bending moments about the middle plane which result from the stresses in steel and concrete on the plane of the crack, we obtain the following equilibrium conditions (per unit length of crack):

$N_{n}=S_{x}\left(C^{2}+\delta_{1} S^{2}\right)+S_{y}\left(S^{2}+\delta_{2} C^{2}\right)+\sigma_{n} a$;

$N_{n t}=\left[S_{y}\left(1-\delta_{2}\right)-S_{x}\left(1-\delta_{1}\right)\right] S C+\sigma_{n t}^{\prime}(a-b)+\sigma_{n t} b$;

$M_{n}=S_{x}\left(C^{2}+\delta_{1} S^{2}\right)\left(d_{x}-\frac{h}{2}\right)+S_{y}\left(S^{2}+\delta_{2} C^{2}\right)\left(d_{y}-\frac{h}{2}\right)-\sigma_{n} a \frac{h-a}{2} ;$

$M_{n t}=-S_{x}\left(1-\delta_{1}\right) S C\left(d_{x}-\frac{h}{2}\right)+S_{y}\left(1-\delta_{2}\right) S C\left(d_{y}-\frac{h}{2}\right)-\sigma_{m t} b \frac{h-b}{2}$

$-\sigma_{m}^{\prime}(a-b) \frac{h-a-b}{2}$

in which $h=$ thickness of the plate (Fig. 1); $\sigma_{n t}^{\prime}=-\sigma_{n t}$ as we assumed; and $d_{x}$, $d_{y}=$ depths to the centroids of bar systems $x$ and $y$ (Fig. 1(c)). We choose $d_{y}$ to refer always to the bar system with the thinner cover, i.e., $d_{x}<d_{y}$.

Substituting Eqs. 2 into Eqs. 4, we obtain a system of four equations which involve seven unknown variables: $S_{x}, S_{y}, \sigma_{n}, \sigma_{n}, a, b$, and $\theta$. To solve these equations, we will obviously need to fix in advance the values of four of these seven variables. The solutions may be carried out for all possible choices of the values of these fixed variables which yield the safe domain.

Since the boundary of the safe domain is nonlinear, anticipation of the shape of the safe domain is helpful for the solution. Similar to the case of in-plane loading solved before $(5,7)$, the safe domain envelopes in the space $\left(S_{x}, S_{y}\right)$ are approximately hyperbolas as sketched in Fig. 2. From this shape, we see that horizontal lines $\left(S_{y}=\right.$ constant) intersect one branch of the hyperbola either in two points or in no point. Thus, if we would fix the value of $S_{x}$ and try to solve for the $S_{y}$ value on the envelope, we would have to expect either two solutions or no solution. This would certainly be an unpleasant circumstance for a numerical solution

The foregoing difficulty can be avoided by a certain substitution of variables. 


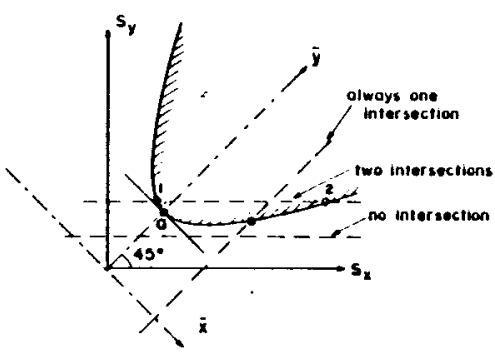

FIG. 2.-Typical Safe Domain Envelope and Rotated Coordinates $\bar{X}, \bar{Y}$

We replace $S_{x}$ and $S_{y}$ by the variables $X=S_{x}-S_{y}$, and $Y=S_{x}+S_{y}$, for which

$S_{x}=\frac{X+Y}{2} ; \quad S_{y}=\frac{Y-X}{2}$

In the $\left(S_{x}, S_{y}\right)$ plane, this substitution means replacing the coordinate axes, $S_{x}$, $S_{y}$, with new coordinate axes rotated by $45^{\circ}$. The new coordinates are given by $\bar{X}=\left(S_{x}-S_{y}\right) / \sqrt{2}=X / \sqrt{2} ; \bar{Y}=\left(S_{x}+S_{y}\right) / \sqrt{2}$. From Fig. 2, we see that if we fix the value of $X$ (or $\bar{X}$ ), the line of constant $\bar{X}$ has always one and only one intersection with the hyperbola. This property greatly facilitates the numerical solution that we outline later.

It will be convenient to introduce further nondimensional parameters, $\kappa, \xi$, and $\eta$, as follows:

$\mathbf{\sigma}_{n t}=-\kappa \sigma_{n} ; \quad \sigma_{n t}^{\prime}=\kappa \sigma_{n}$

$a=\xi h ; \quad b=\eta a$

Substituting now these expressions and Eqs. 5 into Eqs. 4, we obtain the equilibrium relations in the following form:

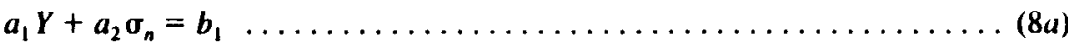

$a_{3} Y+a_{2}(1-2 \eta) \sigma_{n} \kappa=b_{2}$

$a_{4} Y+a_{5} \sigma_{n}=b$

$a_{6} Y-a_{\eta}\left[\xi\left(2 \eta^{2}-1\right)+1-2 \eta\right] \sigma_{n} \kappa=b_{4}$

in which $a_{1}=\frac{1}{2}\left(1+\delta_{1} S^{2}+\delta_{2} C^{2}\right) ; \quad a_{2}=\xi h ; a_{3}=\left(\delta_{1}-\delta_{2}\right) \frac{S C}{2} ;$

$a_{4}=\frac{1}{2}\left[\left(C^{2}+\delta_{1} S^{2}\right)\left(d_{x}-\frac{h}{2}\right)+\left(S^{2}+\delta_{2} C^{2}\right)\left(d_{y}-\frac{h}{2}\right)\right] ; \quad a_{5}=a_{7}(1-\xi) ;$

$a_{6}=\frac{S C}{2}\left[\left(1-\delta_{2}\right)\left(d_{y}-\frac{h}{2}\right)-\left(1-\delta_{1}\right)\left(d_{x}-\frac{h}{2}\right)\right] ; \quad a_{7}=\frac{\xi}{2} h^{2}$;

$b_{1}=N_{n}-\frac{X}{2}\left(C^{2}-S^{2}+\delta_{1} S^{2}-\delta_{2} C^{2}\right) ; \quad b_{2}=N_{n t}+\frac{X}{2}\left(2-\delta_{1}-\delta_{2}\right) S C ;$
$b_{3}=M_{n}-\frac{X}{2}\left[\left(C^{2}+\delta_{1} S^{2}\right)\left(d_{x}-\frac{h}{2}\right)-\left(S^{2}+\delta_{2} C^{2}\right)\left(d_{y}-\frac{h}{2}\right)\right]$

$b_{4}=M_{n t}+\frac{X}{2}\left[\left(1-\delta_{1}\right)\left(d_{x}-\frac{h}{2}\right)+\left(1-\delta_{2}\right)\left(d_{y}-\frac{h}{2}\right)\right] S C$

Now we express $\sigma_{n}$ from Eq. 8a, substitute it into Eq. 8c, and express from it $Y$; then we express $\mathrm{\kappa}$ from Eq. $8 b$, and substitute these expressions into Eq. $8 d$, which provides

$Y=\frac{\xi h b_{3}+a_{5} b_{1}}{\xi h a_{4}+a_{1} a_{5}}$

$\sigma_{n}=\frac{b_{1}-a_{1} Y}{a_{2}}$

$\kappa=\frac{b_{2}-a_{3}}{(1-2 \eta) \sigma_{n} a_{2}}$

$\xi h\left(b_{2}-a_{3} Y\right) \eta^{2}+\left[2 a_{6} Y-h\left(b_{2}-a_{3} Y\right)-2 b_{4}\right] \eta$

$+\left[b_{4}-a_{6} Y+(1-\xi) \frac{h}{2}\left(b_{2}-a_{3} Y\right)\right]=0$

If we now fix the values of $\theta, \xi$, and $X$ as well as $\delta_{1}$ and $\delta_{2}$, we can calculate $Y$ and $\sigma_{n}$ from Eqs. 10a-b. Then we can solve $\eta$ from Eq. 11 and $\kappa$ from Eq. $10 c$. Finally, we calculate the required forces in reinforcement from $\mathrm{Eq}$. 5 , from which we can determine the required cross-section areas of reinforcement as $A_{x}$ $=S_{x} / f_{y}, A_{y}=S_{y} / f_{y}$ in which $f_{y}=$ yield stress of steel.

Note that Eq. 11 is a quadratic equation. When its solutions are complex, they must be rejected as inadmissible (i.e., no equilibrium state exists). When the solutions are real, one of them is always out of the bounds $0 \leq \eta \leq 1$, and so we have at most only one admissible solution.

Not all solutions of Eq. 8 are admissible. The following seven inequalities must be satisfied:

$Y \geq 0 ; \quad \sigma_{n} \leq 0 ; \quad \sigma_{n} \geq-0.85 f_{c}^{\prime} ; \quad \eta \geq 0 ; \quad \eta \leq 1 ; \quad \kappa \leq k ; \quad \kappa \geq-k \ldots$ (12) and these inequalities are sufficient if we neglect the dowel action. We denote here $f_{c}^{\prime}=$ standard 28-day cylinder strength; $0.85 f_{c}^{\prime}=$ effective compression strength for plastic analysis of ultimate loads of beams according to American Concrete Institute (ACI) Code (11); $k=$ friction coefficient of concrete (average value) within the compression zone. The value of $k$ would certainly not be less than the friction coefficient for the cracks in concrete. Based on tests of cracked compressed blocks (25), $k \simeq 1.7$, while the $\mathrm{ACI}$ code recommends $k=1.4$ as a low estimate for the friction of concrete on concrete. For an uncracked compression zone, a somewhat higher value of $k$ may perhaps be applicable. (Note that we mean the secant values of $k$; the tangent values may be considerably smaller.)

If we would take the dowel action into account, four more inequalities would have to be satisfied by the dowel action coefficients $\delta_{1}, \delta_{2}$ :

$\delta_{1} \leq \delta_{0} ; \quad \delta_{1} \geq-\delta_{0} ; \quad \delta_{2} \leq \delta_{0} ; \quad \delta_{2} \geq-\delta_{0}$ 
Here we introduce $\delta_{0}$ as the limiting value of the dowel action coefficient, an empirical quantity.

\section{Solution Algorithm and Safe Degign Doman}

We seek the cross-section areas of reinforcement, or the yield forces, $S_{x}, S_{y}$, for which given internal forces, $N_{x}, \ldots M_{x y}$, can be safely resisted. Equilibrium must be satisfied at a normal crack plane of any orientation, $\theta$. This is our fundamental design hypothesis, analogous to that used before $(5,7)$ for the frictional (slip-free) design of reinforcement for in-plane loading. This hypothesis contrasts with the classical approach in which equilibrium conditions are imposed for only one crack orientation, $\theta$, i.e., the direction normal to the maximum principal stress in concrete.

Safe design is obviously obtained if the reinforcement is not lighter than that required for any crack orientation, $\theta$. Thus, among the solutions for each $\theta$ ( 0 $\leq \theta \leq 180^{\circ}$ ) for a given $X$ we must find the one that maximizes $Y$ (the sum of the yield forces in both reinforcement directions). (Note that when $X$ is fixed an increase of $Y$ represents an increase of both $S_{x}$ and $S_{y}$.)

For each crack direction, the design is safe if there exists at least one admissible equilibrium state. The safety of this assumption is guaranteed by the lower bound theorem of plasticity. This theorem is applicable in our problem as long as our analysis of frictional stresses does not go beyond the equilibrium conditions $(7,18)$, i.e., does not involve kinematics. The kinematics of slip and opening of cracks in concrete normally violates the normality rule of plasticity (6) thereby making the lower bound theorem invalid. In our solution, however, we do not need kinematics and so the application of the lower bound theorem is justified despite friction.

The boundary of the safe design domain is a curve in the space $\left(S_{x}, S_{y}\right)$ which may be described as $Y=F(X)$. The solution of the four equilibrium equations (Eq. 8) is a function of parameters $\theta, \xi$, and $X$, which must be chosen in advance. According to the foregoing considerations, unknown parameters $\theta$ and $\xi$ may be eliminated through the use of the extremizing conditions just derived:

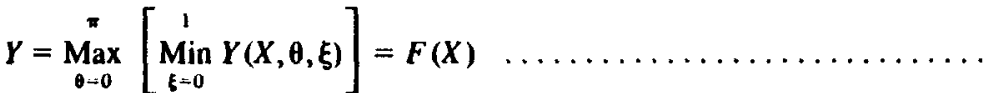

in which the solutions $Y(X, \theta, \xi)$ are the adnissible ones, satisfying all inequalities (Eq. 12). Eq. 14 defines a nonlinear optimization problem.

If we would consider dowel action, we could expand the foregoing condition

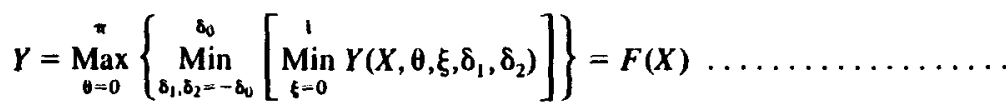

The conditions for maximum and minimum unfortunately cannot be expressed as conditions of a stationary value $(\partial Y / \partial \theta=0, \partial Y / \partial \xi=0)$. The reason: The function $Y(X, \theta, \xi)$ is not smooth and continuous. It exhibits extremes with a discontinuous change of slope, and also extremes at terminal points beyond which no solution exists. This is due to the inequality restrictions (Eq. 12). For instance, over one segment of the curve of $Y$ as a function of $\xi$, coefficient $\kappa$ may vary continuously and remain $\leq k$; this segment may then terminate at a maximum point where $k=k$ beyond which there may exist no solution. In consequence, a numerical solution seems mandatory.

It appears feasible to locate the minimum and maximum simply by scanning the intervals $0 \leq \xi \leq 1$ and $0 \leq \theta \leq 180^{\circ}$. A FØRTRAN IV computer program has been developed for this purpose. The value of $X$ is incremented in discrete steps, $\Delta X$. For each $X$, the minimum of $Y$ is first located by incrementing $\xi$ from 0 to 1 in discrete steps, $\Delta \xi$, and the maximum of these minima of $Y$ is then located by incrementing $\theta$ from $0^{\circ}$ to $180^{\circ}$ in discrete steps, $\Delta \theta$. For each set of values, $X, \xi$, and $\theta$, the solution of $\eta, Y, \sigma_{n}$, and $\kappa$ is found from Eqs. 1112. and the admissibility conditions (Eq. 12) are then checked; if any one of them is violated, the solution is rejected as inadmissible. In computations, the solutions are found inadmissible for a broad range of $\xi$ values.

If no admissible solution exists for some $\theta$ value, one must check first whether this is due to the bending moment at the $\theta$ plane being negative and, thus, calling for reinforcement near the top rather than the bottom surface of the plate. If this is not the case, it means that the plate thickness, $h$, is insufficient to achieve a safe design.

All numerical solutions reported in the sequel have been carried out under the assumption of no dowel action $\left(\delta_{1}=\delta_{2}=0\right)$. In principle, it would not have been much more difficult to scan also the intervals $\delta_{1}, \delta_{2} \in\left(-\delta_{0}, \delta_{0}\right)$, but the computation costs would have become excessive.

To reduce the cost of computation, relatively crude solutions of $\xi, \theta$, and $Y$ have first been obtained using large steps, $\Delta \xi$ and $\Delta \theta$ (e.g., $\Delta \xi=0.05$ and $\Delta \theta$ $=5^{\circ}$ ). The vicinity of the values of $\xi$ and $\theta$, which gave the extremes, was then scanned with smaller steps (e.g., $\Delta \xi=0.005, \Delta \theta=1^{\circ}$ ). This refined scanning near the extremes was done automatically in the program.

It may be of interest that various alternative methods of solution have been tried and were found to be more difficult. For instance, instead of Eq. 8 for $X$, $\sigma_{n}, \eta$, and $k$, one can formulate a system of four equilibrium equations for unknowns, $X, \sigma_{n}, k$, and $\xi$. Then, however, one equation (the one for $\xi$ ) is cubic rather than quadratic. This would be more expensive to solve. Besides, to locate the minimum by scanning the discrete values of $\eta$ instead of $\xi$ appears to be less efficient in this approach.

When $M_{2} / M_{1}<0$, negative bending moments exist on certain planes, and the compression zone is then located at the bottom rather than the top surface of the plate. When the program as just described is run for these crack directions, no admissible equilibrium state is found to be possible. The reason, of course, is that the reinforcement is needed near the top surface. Thus, a complete solution of situations with $M_{2} / M_{1}$ requires running the program also for the situation with interchanged top and bottom (for which $M_{1}$ and $M_{2}$ switch their signs). For such situations, one of course needs reinforcing nets near both surfaces of the plate. Our neglect of possible reinforcement within the compression zone is a simplification which is evidently on the safe side. (A listing of the program, detailed description, user's instructions, and test examples are given in Ref. 3.)

\section{Numeatcal Studies and Analysis of Results}

Numerical examples were solved using various values of friction coefficient, 
$k$. The value $k=1.7$ is probably most realistic. As shown before $(3,6)$, the classical design of reinforced panels under purely in-plane loading $\left(M_{1}=M_{2}\right.$ $=0$ ) is based on the assumption of a through-crack without slip, i.e., without frictional stress which is obtained as the special case for $|k|=k \rightarrow \infty$; this is because $\kappa \rightarrow \infty$ implies that $\sigma_{n}=0$ (since $\left|\sigma_{n}\right|$ cannot be infinite) and that the crack is normal to the direction of the principal tensile stress in concrete $(k \rightarrow$ $\infty$ is represented in computations as $\left.k=10^{6}\right)$. For flexural, as well as predominantly flexural, loading, in which the crack extends through only part of the thickness $(0<a<h)$, our mathematical model corresponds to the classical solution when we set $k \rightarrow \infty$ but not $|\kappa| \rightarrow \infty$, since no limit on shear stress, $\sigma_{n s}$ in the compression zone is imposed in the classical solution. Again, however, instead of examining all possible crack directions, the critical crack direction is assumed to be normal to the principal stress in concrete at the tensile face. In our model, we examine all crack directions.

Safe design boundaries obtained (for $\delta_{0}=0$ ) for six illustrative examples, as defined in Table 1, are plotted in Fig. 3(a-f). Fig. 3(a) shows that the value of the friction coefficient, $k$, can have a large effect. The same is observed in some parts of Figs. $3(b-f)$, but we also see many cases where the effect of friction coefficient is limited or none.

The optimum reinforcement is usually considered as that of minimum weight. This is equivalent to minimizing $Y$, i.e. (for $\delta_{1}=\delta_{2}=\delta_{0}=0$ ):

$Y_{\mathrm{opt}}=\operatorname{Min} F(X)=\operatorname{Min}_{X=-\infty}^{\infty}\left\{\operatorname{Max}_{\theta=0}^{\pi}\left[\operatorname{Min}_{\xi-0}^{1} Y(X, \theta, \xi)\right]\right\}$

Geometrically, the optimum point is obtained as the tangent point of the boundary curve, $F(X)$, and one line, $Y=$ constant (inclined $-45^{\circ}$, see point $a$ in Fig. 2). Each point of the boundary, $Y=F(X)$ (Fig. 2), corresponds to a certain crack direction, $\theta$. For the classical solution, the optinum point is found to correspond always to $\theta=45^{\circ}$. In the present model, the crack angle, $\theta$, for the optimum design may significantly differ from $45^{\circ}$, as the numbers given for the optimum points $\mathrm{a}$ and $\mathrm{b}$ in Fig. $3(a-f)$ indicate.

Comparing Fig. 3(a) with Fig. 3(b-c), which does not involve membrane (inplane) forces, we further observe that the effect of the friction coefficient is more marked when significant membrane forces are present. The examples also differ in the value of ratio $M_{2} / M_{1}$. When it is substantially less than 1.0, large twisting

\begin{tabular}{|c|c|c|c|c|c|c|c|c|c|c|}
\hline $\begin{array}{c}\text { Example } \\
\text { (1) }\end{array}$ & $\begin{array}{c}h, \text { in } \\
\text { inches } \\
\text { (2) } \\
\end{array}$ & $\begin{array}{c}d_{x,} \text { in } \\
\text { inches } \\
\text { (3) }\end{array}$ & $\begin{array}{c}d_{y, \text { in }} \\
\text { inches } \\
\text { (4) }\end{array}$ & $\begin{array}{c}f_{c}^{\prime} \text {, in } \\
\text { pounds per } \\
\text { square inch } \\
\text { (5) }\end{array}$ & $\begin{array}{c}N_{x} \text {, in kips } \\
\text { per inch } \\
\text { (6) }\end{array}$ & $\begin{array}{c}N_{2} / N_{1} \\
(7)\end{array}$ & $\begin{array}{l}a_{N} \\
\text { (8) }\end{array}$ & $\begin{array}{c}M_{x,} \text { in } \\
\text { kips } \\
\text { per } \\
\text { inch } \\
(9) \\
\end{array}$ & $\begin{array}{c}M_{2} / M_{1} \\
(10)\end{array}$ & $\begin{array}{r}\alpha_{M} \\
\text { (11) }\end{array}$ \\
\hline a & $\begin{array}{l}20 \\
20\end{array}$ & $\begin{array}{l}17 \\
17\end{array}$ & $\begin{array}{l}18 \\
18\end{array}$ & $\begin{array}{l}4,000 \\
4,000\end{array}$ & $\begin{array}{c}10 \\
0\end{array}$ & $\begin{array}{l}0.37 \\
0\end{array}$ & $38^{\circ}$ & $\begin{array}{l}200 \\
285\end{array}$ & $\begin{array}{l}0.3 \\
0.8\end{array}$ & $\begin{array}{l}52^{\circ} \\
30^{\circ}\end{array}$ \\
\hline c & 18 & 16 & 16 & 1,500 & 0 & 0 & - & 35 & 0.24 & $53^{\circ}$ \\
\hline d & 20 & 17 & 18 & 4,000 & 13.1 & 0.5 & $30^{\circ}$ & 285 & 0.8 & $30^{\circ}$ \\
\hline c & 20 & 17.5 & 17.5 & 4,000 & 0 & 0 & - & 285 & 0.8 & $30^{\circ}$ \\
\hline f & 20 & 17 & 18 & 4,000 & 14.5 & 0.5 & $15^{\circ}$ & 285 & 0.8 & $30^{\circ}$ \\
\hline
\end{tabular}
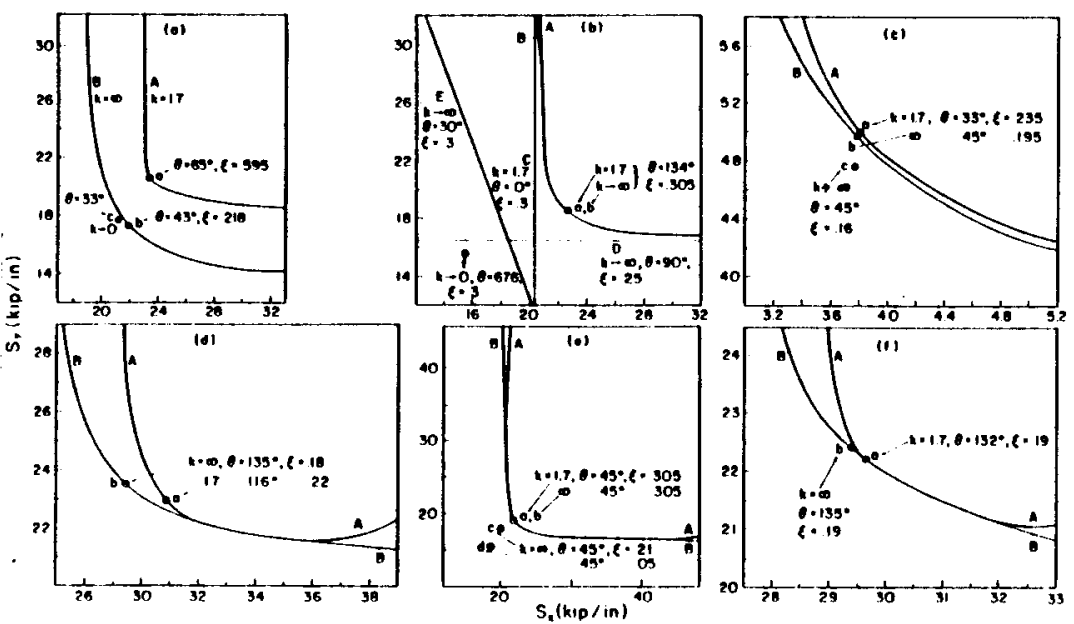

FIG. 3.-Results of Numerlcal Examples Defined in Table 1

moments are present on some planes. Only then can the frictional forces become significant. On the other hand, for $M_{2} / M_{1} \simeq 1$, the effect of friction must be negligible. This is seen when comparing Fig. $3(b-d)$.

One peculiar feature of the frictional (no-slip) design of reinforcement for membrane forces $(5,7)$ is the fact that the safe domain boundary may have a point of horizontal or vertical tangent and reverse its slope beyond that point (increasing $S_{x}$ or $S_{y}$ ). This feature is also discernible in Fig. 3(e). However, as shown in Ref. 7, the boundary segment of reversed slope (i.e., a segment in which both $S_{x}$ and $S_{y}$ increase) is not a true safe domain boundary because addition of one reinforcement cannot make the design unsafe and, thus, cannot pose a demand for increasing the other reinforcement, $S_{y}$. Thus, the boundary segment of reversed slope must be replaced by the horizontal or vertical tangent.

To define the problem in its nondimensional form, we need altogether eighs independent nondimensional input parameters:

$\frac{d_{x}}{h} ; \frac{d_{y}}{h} ; \frac{M_{1}}{f_{c}^{\prime} h^{2}} ; \frac{M_{2}}{M_{1}} ; \alpha_{M} ; \frac{N_{1}}{f_{c}^{\prime} h} ; \frac{N_{2}}{N_{1}} ; \alpha_{N}$

As the nondimensional characteristics of the required reinforcement, it is most convenient to choose

$F_{x}=\frac{S_{x} z_{x}}{M_{1}} ; \quad F_{y}=\frac{S_{y} z_{y}}{M_{1}} \quad$ with $\quad z_{x}=d_{x}-\frac{a}{2} ; \quad z_{y}=d_{y}-\frac{a}{2}$

in which $z_{x}, z_{y}=$ the lever arms between the normal stress resultants in steel and in concrete. The use of $F_{x}$ and $F_{y}$ has the advantage that their value is not too sensitive to the values of $\xi, d_{x} / h, d_{y} / h$, and $f_{c}^{\prime} h^{2} / M$. This would not be true for other nondimensional forms of $S_{x}, S_{y}$, e.g., $S_{x} /\left(f_{c}^{\prime} h\right), S_{y} /\left(f_{c}^{\prime} h\right)$

Since the concrete outside the compression zone plays no role in our mathematical model, the thickness of the plate must actually have no effect. Therefore, 
we could further reduce the number of nondimensional input parameters to seven if we would refer the bending and twisting moments not to the midplane, $h / 2$ but to the plane passing at the middle of the distance, $d_{y}$, to the centroid of the heavier reinforcing system. The seven parameters would be

$\frac{d_{y}}{d_{x}} ; \frac{M_{1}}{f_{c}^{\prime} d_{y}^{2}} ; \frac{M_{2}}{M_{1}} ; \quad \alpha_{M} ; \frac{N_{1}}{f_{c}^{\prime} d_{y}} ; \frac{N_{2}}{N_{1}}$

Referring the moments to a plane other than the midplane would be unconven tional and inconvenient for structural analysis, however.

Example $c$ in Table 1 (Fig. 3(c)) is the same as that solved by Morley (22). and example $e$ the same as that solved by Morlcy (22), Gupta (14), and Bammann (1). The comparison shown in Table 2 reveals that significant differences can arise. Morley and Gupta's solutions can give $7.4 \%$ less reinforcement than the present one, and Baumann's solution can give $18.8 \%$ less reinforcement. These comparisons indicate that the present method is more conservative if the reinforcement directions significantly deviate from the principal stress directions

Baumann (1) presented design charts for the optimum reinforcement under purely flexural loadings $\left(N_{1}=N_{2}=0\right)$ characterized by various $\alpha_{M}$ and $M_{2} / M_{1}$. His charts are shown as the dashed lines in Fig. $4(a-c)$ using the nondimensional variables defined in Eq. 17. For comparison, the same charts were computed with the present method (for $\delta_{0}=0$ ). They are shown as the solid lines in Fig. $4(a-c)$. Further charts of compression resultant, $C_{N}=-a \sigma_{n}$, in concrete are given in Fig. $4(d-e)$

Parameters $\alpha_{M}$ and $M_{2} / M_{1}$ (apart from $N_{1}=N_{2}=\alpha_{N}=0$ ) are not the only nondimensional parameters affecting the solution. The remaining three necessary parameters of Eq. 17, i.e., $d_{x} / h, d_{y} / h$ and $M_{1} /\left(f_{c}^{\prime} h^{2}\right)$ were based on $h=20 \mathrm{in}$. $(508 \mathrm{~mm}), d_{x}=17 \mathrm{in}$. $(431.8 \mathrm{~mm}), d_{y}=18 \mathrm{in} .(457.2 \mathrm{~mm}), f_{r}^{\prime}=4,(00) \mathrm{psi}$ $(27.48 \mathrm{MPa})$, and $M_{1}=300 \mathrm{kip}(1,335 \mathrm{kN})$. Baumann (1) did not need these parameters because he used an approximation in which he avoided solving the depth of the compression zone (or $\xi$ ), estimating $z_{x}$ and $z_{y}$ to be $0.85 d_{m}(0.8-0.9)$ in which $d_{m}=\left(d_{x}+d_{y}\right) / 2$. This allowed him to reduce the problem to that of membrane reinforcement in a tensile layer of the plate which is subjected to membrane normal forces, $M_{1} / z_{x}$ and $M_{2} / z_{y}$, of inclination, $\alpha_{M}$, and is treated independently of the compression zone (for the latter, therefore, no frictional

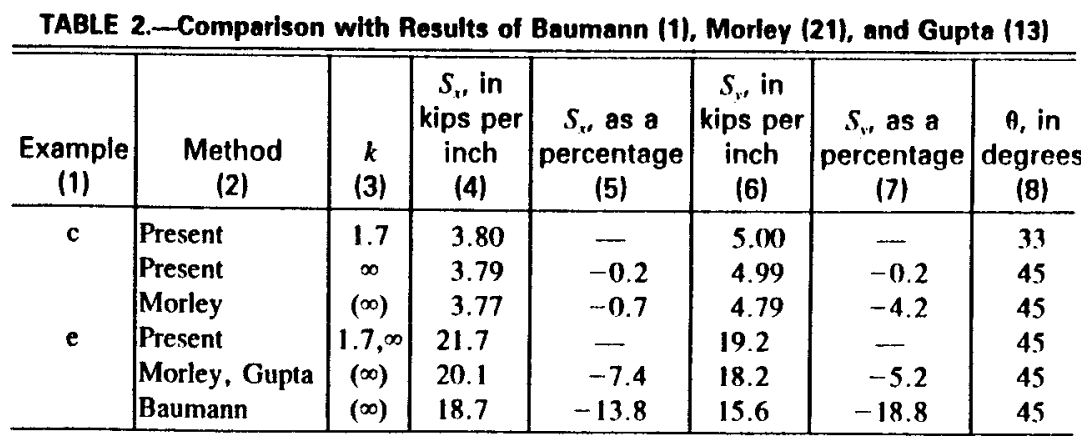

Note: $1 \mathrm{kip} / \mathrm{in} .=175.1 \mathrm{kN} / \mathrm{m}$
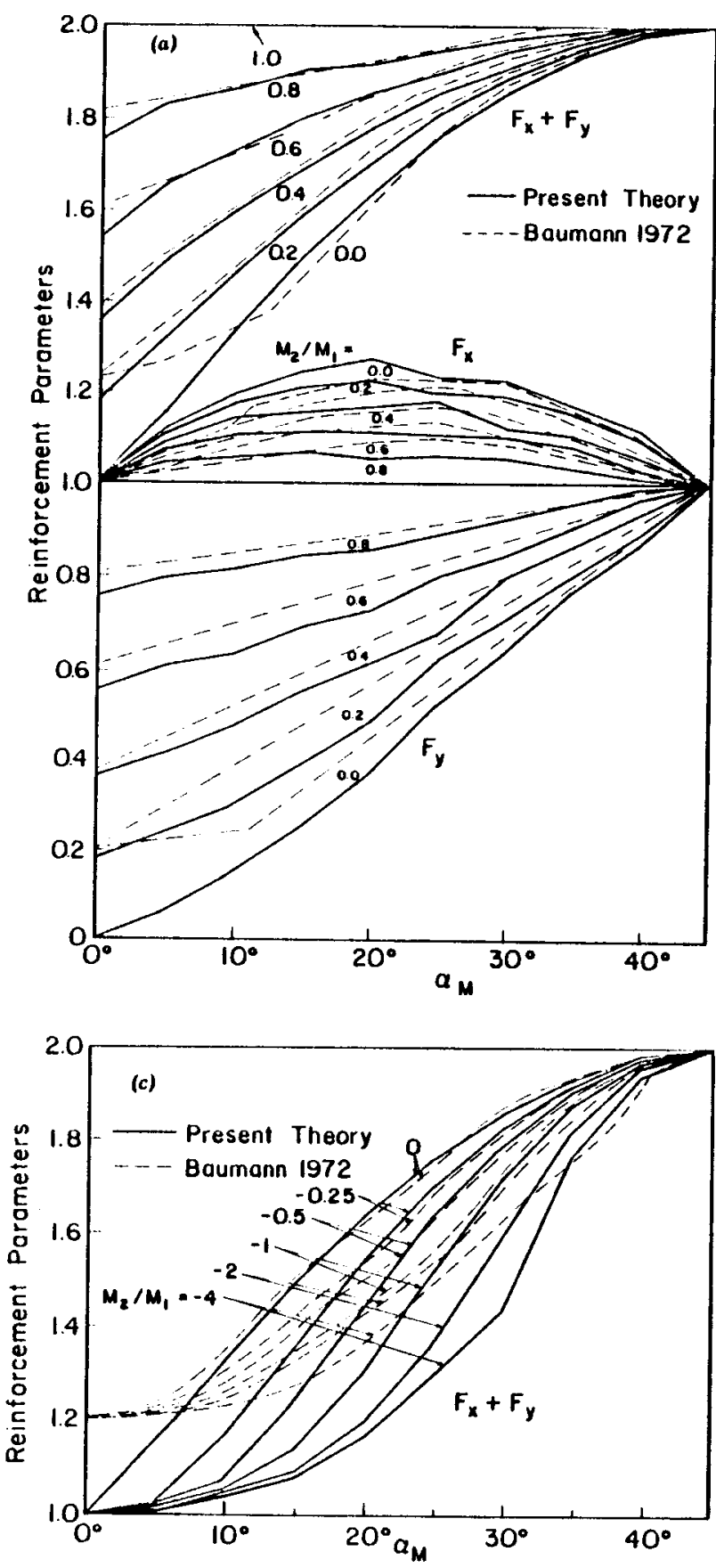

FIG. 4.-Design Charts for Pure Bending 

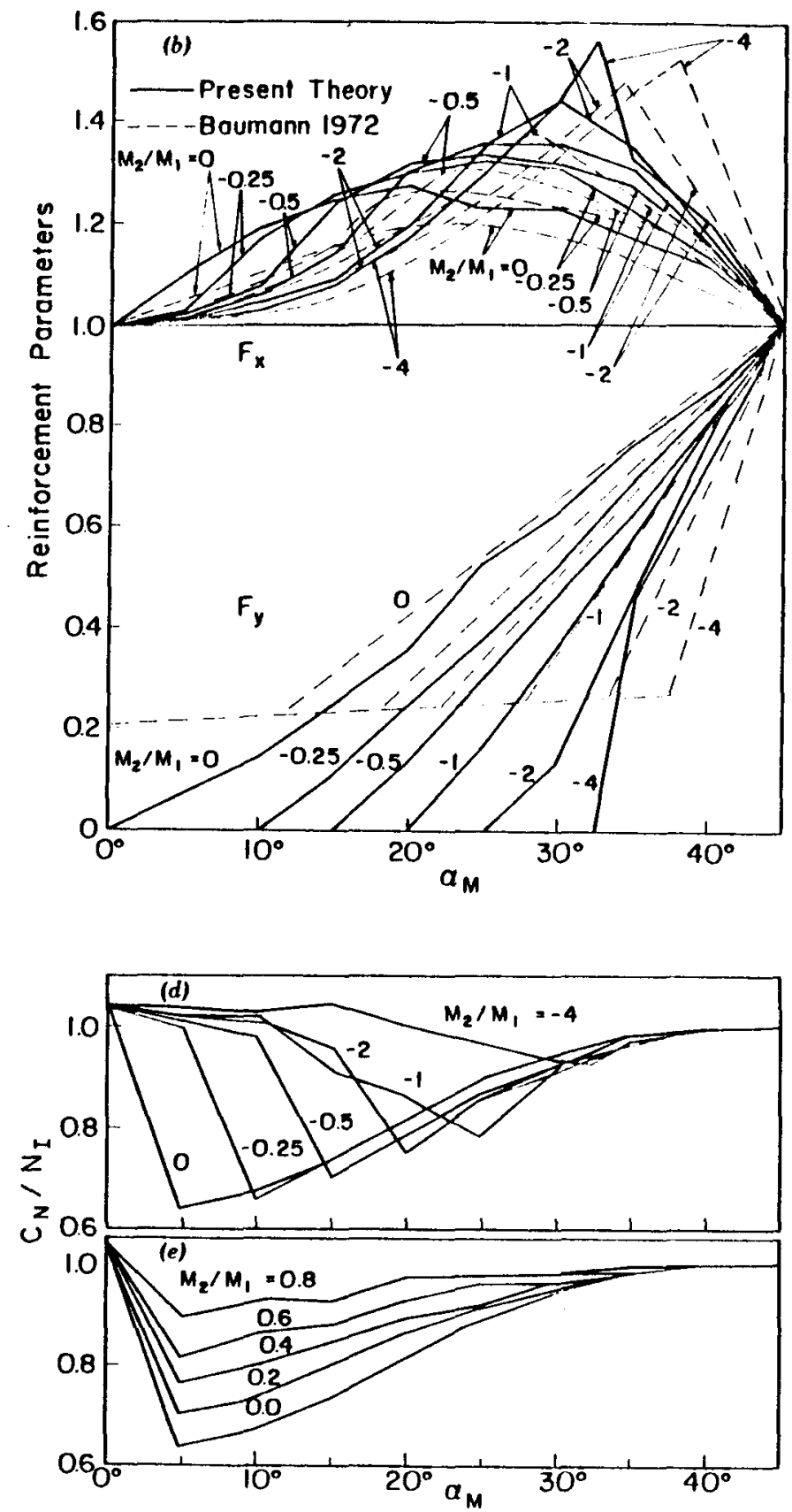

limitations could be imposed). This approximation involves a certain error which partly contributes to the discrepancies seen in Fig. 4(a-c).

Parameters $F_{x}$ and $F_{y}$ (Eq. 17) have the advantage that they are little sensitive to the values of $d_{x} / h, d_{y} / h$, and $M_{1} /\left(f_{c}^{\prime} h^{2}\right)$, while $\xi$ is strongly affected by these parameters. For instance, if we consider $M_{2} / M_{1}=0.8$ and $\alpha_{M}=25^{\circ}$ and reduce $M_{1}$ from 300 to $225 \mathrm{kip}, \xi$ changes from 0.305 to 0.215 while $F_{x}$ changes only from 1.062 to 1.057 , and $F_{y}$ from 0.887 to 0.896 ; if we further reduce $M_{1}$ to $150 \mathrm{kip}, \xi$ changes to 0.140 while $F_{x}$ changes to 1.047 and $F_{y}$ to 0.904 . So Baumann's use of the approximation $Z_{x} \simeq Z_{y} \simeq 0.85 d_{m}$ could not have caused a large error. Moreover, the values of $z_{x}$ and $z_{y}$ obtained in calculating the charts in Fig. 4 were rather close to Baumann's assumption, $z_{x} \simeq z_{y} \simeq 0.85 d_{m} ; z_{x}$ ranged from $0.79 d_{m}$ to $0.85 d_{m}$, and $z_{y}$ from $0.85 d_{m}$ to $0.90 d_{m}$.

Consequently, the main reason for the significant differences between Baumann's and our design charts must lie in the differences in basic assumptions. These are: (1) The crack is not restricted as normal to the principal tensile stress in concrete at the tensile face, but all crack directions, $\theta$, are checked; (2) the horizontal shear stress transmitted in the compression zone is not arbitrary, but is limited by the friction coefficient; and (3) part of the torque can be transmitted by in-plane frictional shear stresses of opposite sign in concrete. The first assumption always causes our design to be more conservative but the other two can lead to a reduction in reinforcement. This explains why the deviations of Baumann's graphs from those in Fig. $3(a-c)$ are on both the plus and minus sides.

Why does this last finding contrast with what was established $(5,7,18)$ for the frictional (no-slip) design under membrane forces, i.e., that the frictional design never leads to less reinforcement than the classical (nonfrictional) design? We would have reached a similar conclusion if we considered crack friction in the tensile layer of the slab. We would then have obtained a precise analog to the previous model $(5,7)$ for membrane loading. For flexural loading, however, the assumption of crack friction in the tensile layer would be questionable unless the assumption of uniform $\sigma_{n}$ (rectangular stress block) were abandoned, as we argued at the outset. Moreover, in contrast to the case of a through-crack, mobilization of friction on the crack would require certain slip movements, which are, however, prevented by the shear stiffness of the compression zone.

\section{Summary and Conclusions}

The limit design of an orthogonal regular plastic reinforcing net in a concrete plate subjected to given nembrane forces and bending and twisting moments is studied. The basic assumptions are: (1) Concrete resists no tension, and a planar normal crack of any angle with the bars may exist in concrete; (2) the normal stresses within the compression zone of the cross section are constant; and (3) the in-plane shear stresses in concrete exist only in the compression zone, are of constant magnitude but possibly of alternating sign, and their ratio to the normal stresses cannot exceed the friction coefficient.

The previously developed frictional (no-slip) limit design for membrane loading is the special case when the compression zone extends over the entire thick ness and the friction stresses are of one sign only. Assumptions (1) and (3) differ 
from the classical approach in which slip on the crack is assumed to be impossible and only a crack of one direction, i.e., that for which there is no friction, is consideted. We drop the classical assumption of an independent tensile layer which is imagined to be under purely membrane loading and which is used for determining the critical crack direction in the classical approach. We compute design charts for reinforcement in the case of no membrane forces and compare them to the classical results of Baumann (1). The main conclusions are:

1. A safe design may be obtained by finding the compression zone depth which gives the minimum reinforcement for any crack angle, and then identifying the maximum of these minima among all crack angles. Next, minimization of the last maximum yields the optimum design. Due to inequality restriclions, these maxima and minima are usually not stationary points. $\Lambda$ computer program can locate these extremes by scanning the complete range of depths and crack angles.

2. While for the pure membrane loading the frictional (no-slip) design never yields less reinforcement than the classical (frictionless) design (1), the same is not true of flexural loading.

3. In terms of the total weight $\left(F_{x}+F_{y}\right.$ in Fig. 4), the differences from the classical design range from $4 \%$ less to $20 \%$ more reinforcement and arc mostly on the plus side. Thus, our design approach is generally more conservative. The differences vanish, however, when the directions of bars, of the membrane principal forces, and of the principal bending moments coincide.

4. While in the classical (frictionless) solution the critical angle between the crack and the bars for the minimum weight design is always $45^{\circ}$; in the present solution this angle may significantly differ from $45^{\circ}$.

5. Some critical states correspond to frictional shear stress distributions in which the shear stress is not constant $(b=a)$ but changes its sign $(b<a)$.

6. For some critical states, the ratio of shear to normal stresses in concrete is equal to the friction limit, and, for others, it is below the friction limit.

7. The conversion of the combined flexural-membrane loading problem to a pure membrane loading problem, as made in the classical approach using the concept of separate tension and compression layers, is an approximation based on a prior guess of the lever arm and on the neglect of the torque that can be transmitted by frictional shear stresses in the compression layer.

8. The solution is greatly facilitated if, instead of the axial forces in the $x$ and $y$ bar systems, their sum and their difference are used as unknowns. This assures that a solution of the equilibrium equations exists and is unique when the dif ferences are fixed.

\section{AcKNomedgmen}

Financial support under National Science Foundation Grant CME8009050 to Northwestern University is gratefully acknowledged. Thanks are also due to $W$ Braestrup of Technical University of Denmark for some valuable critical comments on the original manuscript.

\section{Appendix.-Refenences}

1. Baumann, T., "Zur Frage der Netzbewehrung von Flächentragwerken," Der Bauingenieur, Vol. 47, No. 10, 1971, pp. 367-377.
2. Bazzant, Z. P., and Gambarova, P., "Rough Cracks in Reinforced Concrete," Journal of the Strucural Division, ASCE, Vol. 106, No. ST4, Apr., 1980, Proc. Paper 15330, pp. 819-842 (with Discussion and Closure, 1980, pp. 2579-2581, 1981, 1377-1388)

3. Bažant, Z. P., Lin, C., "Computer Program OPTI for Frictional Limit Design of Reinforcement in Concrete Plates," Report No. 8/-7/665c, Center for Concrete and Geomaterials, Technological Institute, Nonhwestern University, Evanston, Ill., Aug., 1981 .

4. Bažant, Z. P., and Oh, B. H., “Defornation of Net-Reinforced Cracked Concrete Walls," Concrete and Geomaterials Report No. 81-11/665d, Northwestern University, Evanston, Ill., Nov., 1981.

5. Bažant, Z. P., and Tsubaki, T., "Concrete Reinforcing Net: Optimum Slip-Free Limit Design," Journal of the Siructural Division, ASCE, Vol. 105, No. ST2, Proc. Paper 14344, Feb., 1979, pp. 327-346.

6. Bažant, Z. P., and Tsubaki, T., "Slip-Dilatancy Model for Cracked Reinforced Concrele," Journal of the Siructural Division, ASCE, Vol. 106, No. ST9, Proc. Paper 15704, Sept., 1980, pp. 1947-1966.

7. Bażant, Z. P., Tsubaki, T., and Belytschko, T. B., "Concrete Reinforcing Net: Safe Design," Journal of the Siructural Division, ASCE, Vol. 106, No. ST9, Proc. Paper 15705, Scpt., 1980, pp. 1899-19066.

8. Bracstrup, M. W., "Yield Line in Discs, Plates and Shells," Report No. R14, Structural Research Laboratory, Technical University of Denmark, 1970.

9. Braestrup, M. W. "Yield Line Theory and Limit Analysis of Plates and Slabs," Magazine of Concrete Research, Vol. 22, No. 71, June, 1970, pp. 99-106.

10. Brondum-Nielsen, T. "Optimum Design of Reinforced Concrete Shells and Slabs," Report No. R.44. Siructural Research Laboratory, University of Denmark, Copenhagen, 1974, pp. 190-200

11. "Building Code Requirements for Reinforced Concrete," ACl-318-77, ACI Commiltee 318 , American Concrete Institute, Detroit, Mich., 1977.

12. Coukson, P. J., “Generalized Yitld Lines in Reinforced Concrete Slabs," Journa of Structural Mechanics, Vol. 7, No. 1, 1979, pp. 65-82.

13. Cookson, P. J., "A Gieneral Yield Criterion for Orthogonally Reinforced Concrete Fiat Elements," Final Rejont, Colloquium, "Plasticity in Reinforced Concrete," Copenhagen, Demmark, 1979, International Association for Bridge and Structural Enginecting, Vol. 29, Zurich, Swilzerland, pp. 43-50.

14. Gupla, A. K. "Proposed Addition to Proposed ACI Standards: Code Requirements for Nuclear Safety Related Concrete Structures," American Concrete Institute Journal, Proceedings, Vol. 73, No. 7, July, 1976, pp. 431-432.

15. Gupta, A. K., and Sen, S., "Design of Flexural Reinforcement in Concrete Slabs," Journal of the Structural Division, ASCE, Vol. 103, No. ST4, Proc. Paper 12888, Apr., 1977, pp. 793-804.

16. Janas, M., and Sawczuk, A., "Intluence of Positions of Lateral Restraints on Carrying Capacity of Plates," Bulletin No. 58, Commité Eurointernational de Béton, 1966.

17. Atarti, P. "Plastic Analysis of Reinforced Concrete Shear Walls," Introductory Repuit, Colloquium, "Plasticity in Reinforced Concrete," Copenhagen, Denmark, 1979, lincernational Assuciation for Bridge and Structural Engineering, Vol. 28, Zinich, Switcerland, pp. 5160

18. Miati, P., Discussion of "Concrele Reinforcing Net: Safe Design," by Z. P. Bažant, T. 'Tsubuki and T. B. Belytscliko, Journal of the Structural Division, ASCE, Vol. 107, No. ST7, July, 1981, pp. 1391-1983, and closure.

19. Mlarti, P., and Thürlimann, B. "Fliessbedingung für Stahlbeton mit Berücksichtigung der Betonzuglestigkeit," Beton-und Stahlbetonbau, Vol. 72, No. 1, Jan., 1977, pp. 7. 12 .

20. Morley, C. T., "Optimum Reinfurcement of Concrete Slab Elements," The Instiiution of Civil Engineers, Proceedings, Vol. 63, Part 2, June, 1977, pp. 441-454.

21. Morley, C. T., "Optimum Reinforcement of Concrete Slab Elements against Combinations of Moments and Membrane Forces," Magazine of Concrete Research, Vol. 22, No. 72, Sept., 1970, pp. 155-162

22. Morley, C. T., "Skew Reinforcement of Concrete Slabs against Bending and Tor- 
sional Moments," Proceedings of the Institution of Civil Engineers, Vol. 42, Jan., 1969, pp. 57-74

23. Morley, C. T., "Yield Criteria for Elements of Reinforced Concrete Slats," Introductory Report, "Plasticity in Reinforced Concrete," International Assnciation for Bridge and Structural Engineering, Zürich, Switzerland, Report of the Working Commission, Vol. 8, 1979, pp. 35-47.

24. Nielsen, M. P., "The Theory of Plasticity for Reinforced Concrete Slahs," Reports of the Working Commissions, Colloquium International Association for Bridge and Structural Engineering, Copenhagen, Plasticity in Reinforced Concrete, Vol. 28. 1979, pp. 91-114.

25. Paulay, T., and Loeber, P. J., "Shear Transfer by Aggregate Interlock, Shear in Reinforced Concrete," Special Publications SP-42, American Concrete Institute. Detroit, Mich., 1974, pp. 1-15.

26. Rujendran, S., and Morley, C. T., "A General Yield Criterion for Reinforced Concrete Slab Elements," Magazine of Concrete Research, Vol. 26, No. 89, Dec., 1964, pp. 212-220, with Discussion, Dec., 1975. 